\title{
Surfactant protein A suppresses reactive nitrogen intermediates by alveolar macrophages in response to Mycobacterium tuberculosis
}

\author{
Rajamouli Pasula, ${ }^{1}$ Jo Rae Wright, ${ }^{2}$ Diane L. Kachel, ${ }^{1}$ and William J. Martin II ${ }^{1}$ \\ ${ }^{1}$ Division of Pulmonary, Allergy, Critical Care and Occupational Medicine, Department of Internal Medicine, Indiana University \\ School of Medicine, Indianapolis, Indiana 46202-2879, USA \\ ${ }^{2}$ Department of Cell Biology, Duke University Medical Center, Durham, North Carolina 27710, USA \\ Address correspondence to: William J. Martin II, Department of Internal Medicine, Division of Pulmonary, Allergy, Critical Care and \\ Occupational Medicine, Indiana University School of Medicine, 1001 West 10th Street, OPW 425, Indianapolis, Indiana 46202-2879, \\ USA. Phone (317) 630-8445; Fax (317) 630-6386; E-mail: wjmartin@iupui.edu
}

Received for publication February 3, 1998, and accepted in revised form December 22, 1998.

\begin{abstract}
Mycobacterium tuberculosis attaches to, enters, and replicates within alveolar macrophages (AMs). Our previous studies suggest that surfactant protein A (SP-A) can act as a ligand in the attachment of M. tuberculosis to AMs. Reactive nitrogen intermediates (RNIs) play a significant role in the killing of mycobacteria. We have demonstrated that RNI levels generated by AMs were significantly increased when interferon- $\gamma$-primed AMs were incubated with M. tuberculosis. However, the RNI levels were significantly suppressed in the presence of SP-A $(10 \mu \mathrm{g} / \mathrm{ml})$. The specificity of SP-A's effect was demonstrated by the use of $\mathrm{F}\left(\mathrm{ab}^{\prime}\right)_{2}$ fragments of anti-SP-A monoclonal antibodies and by the use of mannosyl-BSA, which blocked the suppression of RNI levels by SP-A. Furthermore, incubation of deglycosylated SP-A with M. tuberculosis failed to suppress RNI by AMs, suggesting that the oligosaccharide component of SP-A, which binds to M. tuberculosis, is necessary for this effect. These results show that SP-A-mediated binding of M. tuberculosis to AMs significantly decreased RNI levels, suggesting that this may be one mechanism by which M. tuberculosis diminishes the cytotoxic response of activated AMs.
\end{abstract}

J. Clin. Invest. 103:483-490 (1999).

\section{Introduction}

Myobacterium tuberculosis remains an important cause of pulmonary infection and is the leading cause of morbidity and mortality worldwide (1-3). After inhalation, $M$. tuberculosis organisms enter alveolar macrophages (AMs), survive and replicate in the host cell, and eventually produce pathogenic effects (4-11). Thus, attachment of $M$. tuberculosis to AMs is likely a necessary requirement for the establishment of the initial tuberculous infection in the alveolar spaces. However, much remains unknown about the mechanisms by which $M$. tuberculosis survive and replicate inside AMs.

Several studies have suggested the involvement of multiple receptors (CR1, CR3, mannose receptor, transferrin receptor, CD14, C2a component of complement, and an unknown receptor that is inhibited by $\beta$-glucan) on the surface of macrophages that mediate the binding and phagocytosis of $M$. tuberculosis organisms (12-18). All of these receptors have been implicated as potential mediators of attachment of M. tuberculosis. The diversity of entry mechanisms suggests that the route of entry may influence the fate of ingested tubercle bacilli in AMs (19).

Macrophages have the ability to recognize and kill invading microorganisms (20). After inhalation, $M$. tuberculosis are rapidly ingested by AMs. Ingestion of $M$. tuberculosis by AMs leads to the secretion of cytokines that subsequently affect the intracellular survival of mycobacteria (21). Activation of macrophages has been identified as important for controlling growth of the microorganisms. Activated macrophages produce reactive nitrogen intermediates (RNIs) that are highly toxic to various intracellular pathogens (22). These include RNIs produced by the nitric oxide synthase/L-arginine-dependent pathway in macrophages and are thought to represent a major killing mechanism of mycobacteria in vitro (23-25). Administration of $M$. tuberculosis in vivo induces production of RNI by rat AMs (26). Furthermore, nitric oxide synthase knockout mice are highly susceptible to $M$. tuberculosis infection (27).

Surfactant protein A (SP-A) has a multifunctional role in the lung (28). SP-A, the major protein component of surfactant, is a C-type lectin and contains a region on the molecule known as the carbohydrate recognition domain $(29,30)$. The carbohydrate recognition domain shares several structural features with complement factor $\mathrm{C} 1 \mathrm{q}$ and mannose-binding protein. SP-A functions as a nonimmune opsonin for a variety of bacterial pathogens and viruses (31-34). It is also thought that SP-A plays an important role in the modulation of the inflammatory and immunological response (35). Recent studies suggest that SP-A alters oxygen radical production $(36,37)$ and blocks the costimulatory signals crucial for in vitro T-lymphocyte cell activation (38). AMs incubated with SP-A have a decrease in superoxide production, indicating a dampening of the respiratory burst $(36,38)$ and suggesting that SP-A has a protective role against the oxidant injury caused by AMs in the lung. 
Others, however, have found SP-A to stimulate the respiratory burst of AMs $(39,40)$. The reasons for these different findings are not completely understood but may be related to different methods used to purify SP-A.

Subjects with HIV are at increased risk for tuberculosis even before there is significant depletion of $\mathrm{CD}^{+}$ lymphocytes (41). A recent study conducted by our laboratory indicates that bronchoalveolar lavage (BAL) fluid from HIV-infected individuals increases attachment of $M$. tuberculosis to AMs (42). The factor in the lavage fluid that increased $M$. tuberculosis attachment is SP-A. However, it is not clear how SP-A might affect the survival or growth of M. tuberculosis within AMs.

To determine possible underlying mechanisms, we have examined the production of RNI by interferon- $\gamma($ IFN- $\gamma$ ) -activated murine AMs in response to $M$. tuberculosis and have demonstrated that SP-A-mediated attachment of $M$. tuberculosis to AMs inhibited RNI production by AMs. This RNI inhibitory effect of SP-A was reversed significantly by the addition of anti-SP-A antibody or mannosyl-BSA. Furthermore, deglycosylated SP-A did not have a significant effect on RNI production, suggesting that the oligosaccharide component of SP-A is necessary for this inhibitory effect. Finally, in addition to inhibiting RNI production, SP-A-mediated attachment was associated with enhanced growth of $M$. tuberculosis in AMs, suggesting one possible mechanism by which the mycobacteria may have enhanced survival.

\section{Methods}

M. tuberculosis isolation. The H37Ra strain of M. tuberculosis (American Type Culture Collection, Rockville, Maryland, USA) was cultured at $37^{\circ} \mathrm{C}$ in a $5 \% \mathrm{CO}_{2}$ atmosphere in dispersed form in Middlebrook $7 \mathrm{H} 9$ broth (Difco Laboratories, Detroit, Michigan, USA) containing albumin, dextrose, and catalase as enrichments. Bacterial cultures 10-14 days old were centrifuged, washed once with saline, and the final concentration of the bacterial suspension was adjusted to $10^{6}$ organisms $/ \mathrm{ml}$. Bacterial concentration was determined using a Spectronic 20D spectrophotometer (43) (Milton Roy Co., Rochester, New York). To achieve a single cell suspension, the mycobacterial suspension was briefly sonicated $(20 \mathrm{~W}$ for 5-10 s). Then the suspension was gently agitated and allowed to stand for $5 \mathrm{~min}$. The top portion of the suspension containing bacilli was used in the assay. Each batch of the bacterial suspension was stained with Kinyoun stain (Midatlantic Biomedical Inc.,
Paulsboro, New Jersey, USA) and observed under a microscope to check the purity of the suspension. Routinely, samples of bacteria were also grown on 7H11 Middlebrook medium (Difco Laboratories) and maintained as stock.

SP-A isolation and purification. Human SP-A was isolated according to Wright et al. (44). Briefly, a surfactant pellet was obtained by centrifugation $(10,000 \mathrm{~g}$ for $60 \mathrm{~min})$ of BAL fluid from patients with alveolar proteinosis then extracted with butanol. The butanol-insoluble proteins were resuspended in octylglucoside to solubilize serum proteins. SP-A purified by this method was dialyzed extensively against $5 \mathrm{mM}$ Tris buffer ( $\mathrm{pH}$ 7.4), then quantified by the Lowry protein assay (45). SP-A purity was verified by SDS-PAGE (46). Deglycosylated SP-A was prepared according to the method of Gaynor et al. (47).

Rat SP-A was isolated according to Kuroki et al. (48), with minor modifications. BAL was obtained from rats that had received intratracheal injection of silica $(0.25 \mathrm{mg} / \mathrm{rat}) 4$ weeks before sacrifice. The lavage was centrifuged at 22,000 $\mathrm{g}$ for $18 \mathrm{~h}$. The pellet ( $4 \mathrm{ml}$ from 10 rats) was injected into $200 \mathrm{ml}$ of butanol and stirred for $30 \mathrm{~min}$. The butanol extract was centrifuged at $15,000 \mathrm{~g}$ for $30 \mathrm{~min}$. The pellet was resuspended in butanol, centrifuged, and finally resuspended in $5 \mathrm{mM}$ Tris buffer ( $\mathrm{pH}$ 7.4) and dialyzed against the same buffer. Insoluble material was removed by centrifugation at $60,000 \mathrm{~g}$ for $1 \mathrm{~h}$. The supernatant was brought up to $10 \mathrm{mM} \mathrm{CaCl}$, passed through a fucoseagarose column (Sigma Chemical Co., St. Louis, Missouri, USA), and washed with approximately $10 \mathrm{ml}$ of $5 \mathrm{mM}$ Tris containing $10 \mathrm{mM} \mathrm{CaCl}_{2}(\mathrm{pH} 7.8$ ). Calcium-dependent fucose-binding proteins were eluted with $5 \mathrm{mM}$ Tris, $2 \mathrm{mM}$ EDTA. SP-A stocks were incubated with polymixin agarose to reduce endotoxin contamination to undetectable levels (49). Human SP-A was used in most experiments to determine the effects of SP-A on AM response to M. tuberculosis; rat SP-A was used in select studies to verify that the effects were not simply species specific.

Production of murine monoclonal antibodies to buman SP-A. Murine anti-human SP-A monoclonal antibodies (MABs) were generated according to the method of Kohler and Milstein (50), with minor modifications. Briefly, BALB/c mice were immunized with $100 \mu \mathrm{g}$ of purified SP-A emulsified in Hunter's Titermax adjuvant (CytRx Corp., Norcross, Georgia, USA) on days 0 and 14. A booster injection without the adjuvant was given on day 28. Three days after the booster injection, splenocytes harvested from the immune spleen were mixed with mouse myeloma cell lines SP2/O. Ag.14 (American Type Culture Collection) and fused in the presence of $1 \mathrm{ml}$ of $50 \%$ polyethylene glycol (Sigma Chemical Co.) for $1 \mathrm{~min}$. After removing the fusing agent by washing with RPMI-1640 (Sigma Chemical Co.), the hybridoma cells were resuspended in hypoxanthine, aminopterin, and thymidine selec-

\section{Figure 1}

Effect of IFN- $\gamma$ alone and IFN- $\gamma$ and $M$. tuberculosis on RNI production by AMs. AMs were stimulated with increasing concentrations of IFN- $\gamma$ alone $(0-1,000 \mathrm{U} / \mathrm{ml})$ for $48 \mathrm{~h}$ or with IFN- $\gamma$ for $48 \mathrm{~h}$ with the addition of $M$. tuberculosis after $24 \mathrm{~h}$ IFN- $\gamma$ preincubation. After incubation, the amount of RNI present in the supernatants was determined by IFN- $\gamma$ alone and was minimal in comparison to the RNI produced by IFN- $\gamma$-stimulated AMs in the presence of $M$. tuberculosis infection. The production of RNI occurred in a concentration-dependent manner, reaching a maximum at $100 \mathrm{U}$ IFN$\gamma / \mathrm{ml}$. Results are expressed as mean \pm SEM of three experiments performed in triplicate. AMs, alveolar macrophages; IFN- $\gamma$, interferon- $\gamma ;$ MTB, M. tuberculosis; $R N I$, reactive nitrogen intermediates.

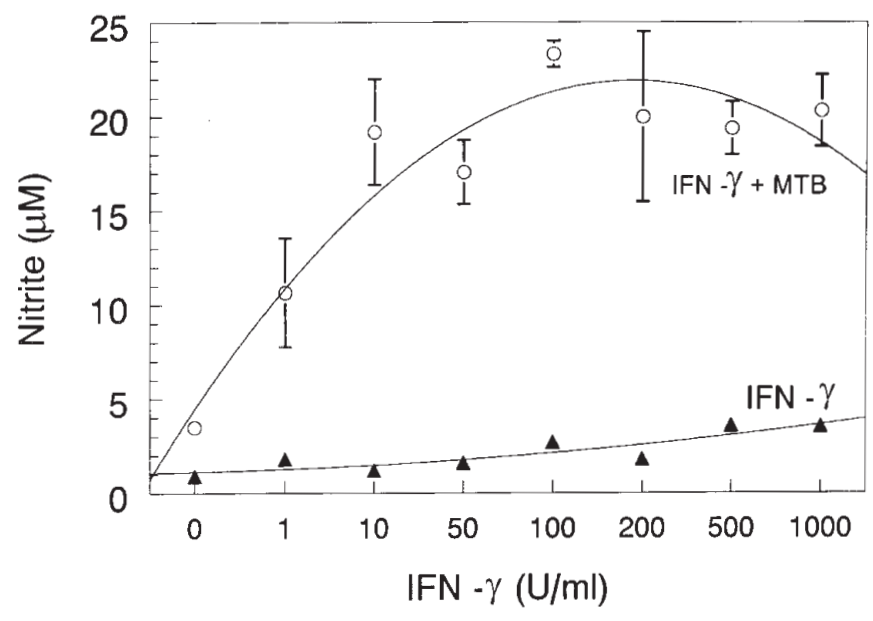


tive media containing $10 \% \mathrm{FCS}$, glutamine $(300 \mu \mathrm{g} / \mathrm{ml})$, penicillin $(100 \mathrm{U} / \mathrm{ml})$, streptomycin $(100 \mu \mathrm{g} / \mathrm{ml})$, gentamycin $(40 \mu \mathrm{g} / \mathrm{ml})$, and nystatin $(25 \mathrm{IU} / \mathrm{ml}$ ) (Sigma Chemical Co.). The hybridoma cells were plated into 24 -well tissue culture plates with a concentration of $10^{5}$ cells $/ \mathrm{ml}$ and incubated for 2 weeks at $37^{\circ} \mathrm{C}$ in $5 \%$ $\mathrm{CO}_{2}$. After 2 weeks, the culture supernatants were tested for the presence of anti-SP-A antibodies by ELISA. Cultures containing antibodies were cloned by limiting dilution into 96 -well tissue culture plates containing feeder cells (51). The positive clones were recloned until the clonal efficiency approached monoclonality. After again screening for the presence of antibodies by ELISA, these MABs were confirmed by Western blotting. The positive clones that secreted antibodies were expanded in the ascites of pristane- (Sigma Chemical Co.) injected BALB/c mice. MABs from the ascites were purified by MAPS II kit (Bio-Rad Laboratories Inc., Hercules, California, USA) and quantified by BCA protein assay (Pierce Chemical Co., Rockford, Illinois, USA). The F(ab') fragments were produced according to the manufacturer's directions (Bio-Rad Laboratories Inc.).

Interaction of AMs with M. tuberculosis. Ten-week-old Swiss-Webster pathogen-free mice were purchased from Harlan Sprague Dawley (Indianapolis, Indiana, USA). Murine AMs were isolated by BAL as described previously (52). Briefly, the mice were sacrificed by intraperitoneal injection of Beuthanasia-D solution (Schering-Plough Animal Health Corp., Kenilworth, New Jersey, USA). The trachea was cannulated after a midline neck incision, and the lungs lavaged 10 times with $1.0 \mathrm{ml}$ of $0.9 \%$ sodium chloride solution containing $0.6 \mathrm{mM}$ EDTA, penicillin $100 \mathrm{U} / \mathrm{ml}$, gentamicin $40 \mu \mathrm{g} / \mathrm{ml}$, and amphotericin B $0.5 \mu \mathrm{g} / \mathrm{ml}$. Approximately 7-8 $\mathrm{ml}$ of lavage fluid was obtained from each mouse. AMs were separated from the lavage fluid by centrifugation at $600 \mathrm{~g}$ for $10 \mathrm{~min}$, washed three times with normal saline, and resuspended in DMEM (BioWhittaker Inc., Walkersville, Maryland, USA) with glutamine $300 \mu \mathrm{g} / \mathrm{ml}, \mathrm{NaHCO}_{3}$, and $20 \mathrm{mM}$ HEPES ( $\mathrm{pH}$ 7.2). Preparations were demonstrated to contain $98 \%$ AMs by routine Diff-Quick (Herleco, Aguada, Puerto Rico) staining and to be $>95 \%$ viable by trypan blue exclusion. AMs were plated at a density of $2.5 \times 10^{5} / 100 \mu \mathrm{l} /$ well in 96-well tissue culture plates in an antibiotic-free DMEM. All assays were conducted at $37^{\circ} \mathrm{C}$ in a $5 \% \mathrm{CO}_{2}$ atmosphere.

AMs were primed with or without murine IFN- $\gamma$ (GIBCO BRL, Grand Island, New York, USA) for $24 \mathrm{~h}$ at $37^{\circ} \mathrm{C}$ in a $5 \% \mathrm{CO}_{2}$ atmosphere. Freshly isolated $M$. tuberculosis organisms were added to adherent AMs in 96-well plates in the ratio of 10:1, and the cultures were incubated for $2 \mathrm{~h}$. After the infection, the AMs were washed to remove any unbound organisms. Fresh medium containing IFN- $\gamma$ was then added and incubated for various time intervals.

To determine the optimal concentration of IFN- $\gamma$, AMs were incubated in the presence of IFN- $\gamma(0-1,000 \mathrm{U}$ IFN- $\gamma / \mathrm{ml})$ for 48 $h$. After the incubation, the cell-free media was tested to determine the amount of RNI accumulated as described below.

Measurement of RNIs. Nitric oxide production was assessed by measuring nitrite. The amount of nitrite formed from RNI was determined from the cell-free supernatants of AMs and M. tuberculosis-infected cultures by the Griess reagent according to Green et al. (53). Briefly, $50 \mu \mathrm{l}$ of the cell-free supernatant was mixed with $50 \mu \mathrm{l}$ of freshly prepared Griess reagent (1\% sulfanilamide, $0.1 \%$ naphthylethylene-diamide-dihydrochloride, and $2.5 \% \mathrm{H}_{3} \mathrm{PO}_{4}$ ) and incubated for $10 \mathrm{~min}$ at room temperature. After the incubation, the plate was read at $540 \mathrm{~nm}$ in an automatic Titertek Multiscan ELISA reader (Flow Laboratories, McLean, Virginia, USA). Nitrite concentrations were determined from a standard curve using sodium nitrite $(0-500 \mu \mathrm{M})$. The results are expressed as $\mu \mathrm{mol}$ nitrite. Cell viability of AMs after incubation with $M$. tuberculosis was determined at various time intervals after infecting AMs with M. tuberculosis by the trypan blue exclusion method. The RNI assay was determined using AM cultures with $M$. tuberculosis alone, in the presence and

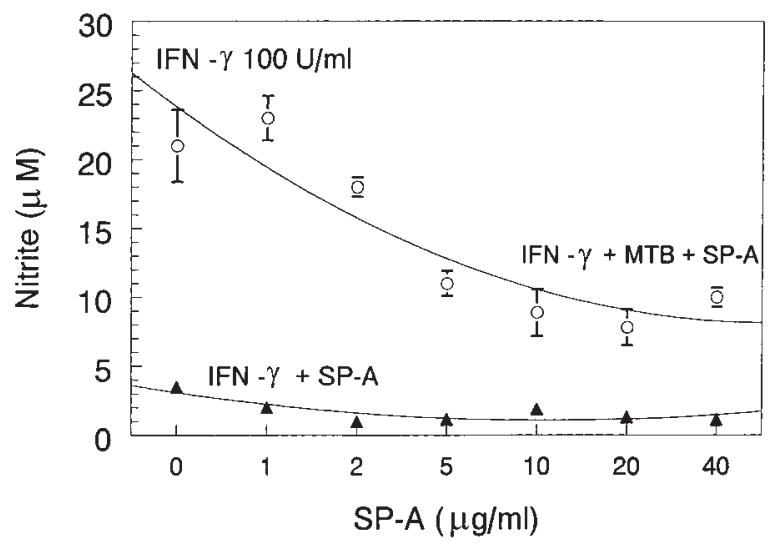

Figure 2

Effect of SP-A concentrations on RNI production by IFN- $\gamma$-stimulated $\mathrm{AMs}$ in the presence and absence of $M$. tuberculosis. AMs were stimulated with IFN- $\gamma(100 \mathrm{U} / \mathrm{ml})$ for $48 \mathrm{~h}$ in the presence of increasing concentrations of exogenous human SP-A $(0-40 \mu \mathrm{g} / \mathrm{ml})$ at $37^{\circ} \mathrm{C}$. Additional AMs were stimulated with IFN- $\gamma$ for $24 \mathrm{~h}$ in the presence of increasing concentrations of exogenous human SP-A $(0-40 \mu \mathrm{g} / \mathrm{ml})$ at $37^{\circ} \mathrm{C}$ and then infected with $M$. tuberculosis for an additional $24 \mathrm{~h}$. After incubation, the supernatants were collected and RNI levels were determined. The results indicate that concentrations of SP-A up to $20 \mu \mathrm{g} / \mathrm{ml}$ significantly decreased nitrite production by IFN- $\gamma$-stimulated AMs infected with $M$. tuberculosis. Results are expressed as mean \pm SEM of three experiments performed in triplicate. SP-A, surfactant protein A.

absence of SP-A $(0-40 \mu \mathrm{g} / \mathrm{ml})$, IFN- $\gamma(100 \mathrm{U} / \mathrm{ml})$, and lipopolysaccharide (LPS) $(10 \mu \mathrm{g} / \mathrm{ml})$.

To determine the possible mechanisms involved in SP-A modulation of RNI production, the assay was performed in the presence or absence of $\alpha$-methylene-D-mannosepyranidose (mannosyl-BSA) $(1,000 \mu \mathrm{g}$ with a molar ratio of mannose/albumin of $26: 1)$, and $F\left(\mathrm{ab}^{\prime}\right)_{2}$ fragments of anti-human SP-A MABs $(100 \mu \mathrm{g} / \mathrm{ml})$, heat-inactivated SP-A $\left(100^{\circ} \mathrm{C}\right.$ for $\left.10 \mathrm{~min}\right)$, and deglycosylated SP-A alone $(10 \mu \mathrm{g} / \mathrm{ml})$.

Additional experiments were conducted to determine whether SP-A scavenges RNI products. SP-A $(10 \mu \mathrm{g} / \mathrm{ml})$ was incubated with increasing concentrations of sodium nitrite $\left(\mathrm{NaNO}_{3}\right)$ from $1 \mu \mathrm{M}$ to $500 \mu \mathrm{M}$ for $24 \mathrm{~h}$ at $37^{\circ} \mathrm{C}$. After the incubation, the amount of RNI that accumulated in the supernatant in the presence or absence of SP-A was determined using the Griess reagent.

Endotoxin assay. The amount of endotoxin present in all the reagents (media, buffers, bacterial cultures, IFN- $\gamma$, SP-A) was assayed by the quantitative chromogenic Limulus amoebocyte lysate assay (BioWhittaker Inc.) in the presence or absence of 100 U polymyxin B/ml (Calbiochem-Novabiochem Corp., San Diego, California, USA). Endotoxin concentrations present in the unknown samples were determined from the standard curve using known endotoxin concentrations $(0-500 \mu \mathrm{M})$. The sensitivity of the assay is $<1 \mathrm{ng} / \mathrm{ml}$. Only endotoxin-free SP-A and reagents were used in this study. In addition, all the experiments were conducted in the presence or absence of polymyxin $\mathrm{B}(100 \mathrm{U} / \mathrm{ml})$, which has been shown to completely inhibit endotoxin function (54).

Growth of $M$. tuberculosis determined by radiometric BACTEC method. The growth of M. tuberculosis within AMs was determined using a radiometric BACTEC 460 TB system (Becton Dickinson Diagnostic Instrument Systems, Sparks, Maryland, USA) $(55,56)$. The accuracy of the BACTEC method was confirmed with colony-forming units, a standard method to measure M. tuberculosis growth. The BACTEC method is based on use of ${ }^{14} \mathrm{C}$ substrate present in the medium by mycobacteria and subsequent release of ${ }^{14} \mathrm{CO}_{2}$ into the atmosphere above the medium. Briefly, AMs were infected with M. tuberculosis at a 
$a$

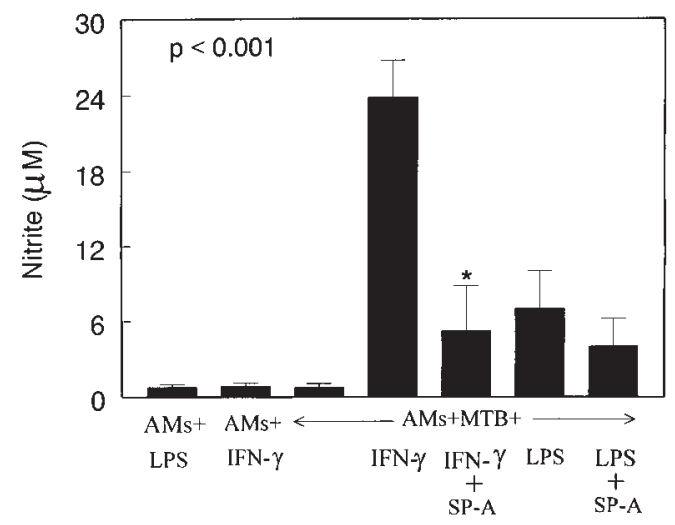

$\boldsymbol{b}$

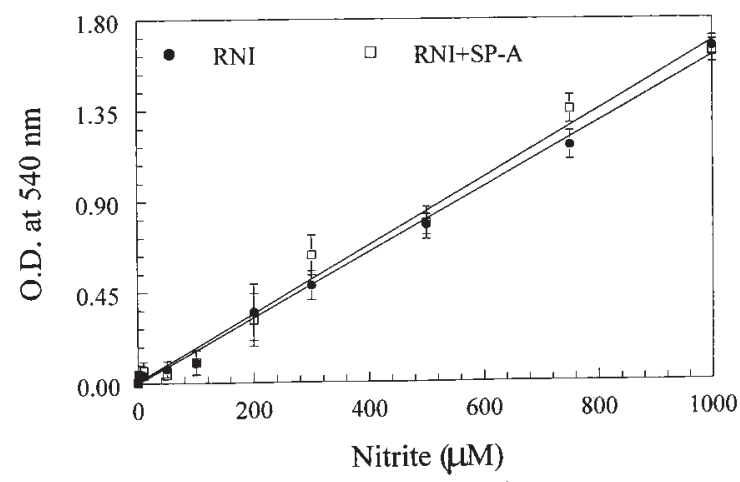

Figure 3

Effect of SP-A on AM generation of RNIs. (a) AMs were incubated with M. tuberculosis alone, and in presence or absence of IFN- $\gamma(100 \mathrm{U} / \mathrm{ml}), \mathrm{SP}-\mathrm{A}$ $(5 \mu \mathrm{g} / \mathrm{ml})$, or LPS $(10 \mu \mathrm{g} / \mathrm{ml})$. These results suggest that SP-A significantly decreased RNI production induced by IFN- $\gamma(P<0.001)$; however, addition of SP-A to LPS-primed AMs did not significantly decrease RNI levels $(P>0.05)$. (b) SP-A $(10 \mu \mathrm{g} / \mathrm{ml})$ was incubated with increasing concentrations of sodium nitrite $\left(\mathrm{NaNO}_{3}\right)$ from $1 \mu \mathrm{M}$ to $500 \mu \mathrm{M}$ for $24 \mathrm{~h}$ at $37^{\circ} \mathrm{C}$. After the incubation, the amount of RNI that accumulated in the supernatant was determined using the Griess reagent. The RNI levels increased in an $\mathrm{NaNO}_{3}$ concentration-dependent manner, and there was no significant effect of SP-A on RNI determination. Thus, there is no evidence that SP-A directly scavenges $\mathrm{NaNO}_{3}$. Results are expressed as mean \pm SEM of three experiments performed in triplicate. LPS, lipopolysaccharide.

ratio of 1:10 (AMs/organisms). After incubation, AMs infected with M. tuberculosis organisms were washed with DMEM to remove unbound $M$. tuberculosis organisms. The AMs were lysed by the addition of $50 \mu \mathrm{l}$ of $0.25 \%$ SDS per well for $10 \mathrm{~min}$. After adding $20 \mu \mathrm{l} 20 \%$ BSA to neutralize the effect of SDS, the total volume was brought up to $0.5 \mathrm{ml}$ with saline and inoculated into BACTEC 12 Middlebrook vials containing $0.1 \mathrm{ml}$ PANTA, an antibiotic supplement (Becton Dickinson Diagnostic Instrument Systems). The vials were incubated at $37^{\circ} \mathrm{C}$, with growth of $M$. tuberculosis being determined every $24 \mathrm{~h}$ by a 460 BACTEC TB instrument (Becton Dickinson Diagnostic Instrument Systems) and expressed as a growth index from 0-999, with an index $>10$ indicating significant growth.

To determine if SP-A would modulate nitrite-mediated killing of M. tuberculosis in the absence of AMs, $\mathrm{NaNO}_{3}(100 \mu \mathrm{M})$ was incubated with $M$. tuberculosis in the presence or absence of SP-A (5 $\mu \mathrm{g} / \mathrm{ml}$ ) for $24 \mathrm{~h}$ at $37^{\circ} \mathrm{C}$. After the incubation, the whole reaction mixture was made up to $0.5 \mathrm{ml}$ and inoculated into the BACTEC vials. After a further $24 \mathrm{~h}$ incubation at $37^{\circ} \mathrm{C}$, the growth of $M$. tuberculosis was determined every $24 \mathrm{~h}$ by the BACTEC method.

Statistical analysis. All experiments were conducted in triplicate, and data are expressed as the mean \pm SEM. Data were compared using Student's $t$ test or ANOVA with paired comparisons with significance at $P<0.05(57)$.

\section{Results}

Effect of IFN- $\gamma$ on RNI production by AMs. AMs were treated with various concentrations of IFN- $\gamma$ for 48 hours at $37^{\circ} \mathrm{C}$. RNI production increased as the concentrations of IFN- $\gamma$ increased, with maximum production occurring at $100 \mathrm{U} / \mathrm{ml}$ (Fig. 1). No significant RNI levels were detected with media and IFN- $\gamma$ alone $(0.16 \pm 0.9 \mu \mathrm{M})$. AMs in the presence of M. tuberculosis alone produced 4.5 $\pm 2.5 \mu \mathrm{M}$ of RNI. When AMs were primed with various concentrations of IFN- $\gamma$ for 24 hours and subsequently incubated with $M$. tuberculosis for an additional 24 hours at $37^{\circ} \mathrm{C}$, RNI production was further increased compared with AMs alone (Fig. 1). Therefore, on the basis of these results, all subsequent experiments with AMs were incubated with $100 \mathrm{U} / \mathrm{ml}$ of IFN- $\gamma$.

Effect of SP-A concentrations on RNI production by AMs. To determine the effect of SP-A on IFN- $\gamma$-primed AMs, AMs were incubated with IFN- $\gamma(100 \mathrm{U} / \mathrm{ml})$ and various SP-A concentrations $(0-40 \mu \mathrm{g} / \mathrm{ml})$. RNI production was decreased by SP-A in a concentration-dependent manner (Fig. 2), with maximal inhibition occurring at $10 \mu \mathrm{g} / \mathrm{ml} \mathrm{SP-A}(P<0.001)$. Similarly, RNI

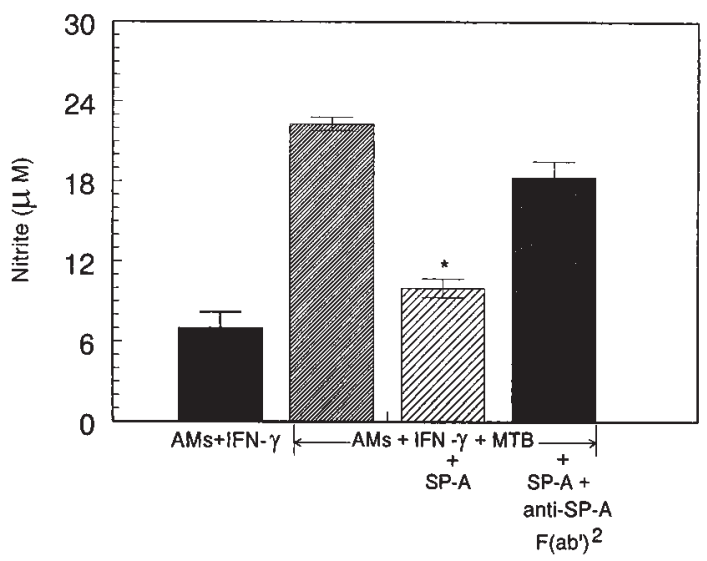

\section{Figure 4}

Effect of anti-SP-A F $\left(a b^{\prime}\right)_{2}$ fragments on SP-A reduction of RNI production by IFN- $\gamma$-stimulated AMs infected with $M$. tuberculosis. Preincubation of IFN- $\gamma$-primed AMs and M. tuberculosis with SP-A significantly decreased RNI production by the infected AMs, whereas addition of 100 $\mu \mathrm{g} / \mathrm{ml}$ of anti-SP-A $\mathrm{F}\left(\mathrm{ab} \mathrm{b}^{\prime}\right)_{2}$ fragments significantly blocked the effect of $\mathrm{SP}-\mathrm{A}$ on RNI production. Results are expressed as mean $\pm \mathrm{SEM}$ of three experiments performed in triplicate. 
a

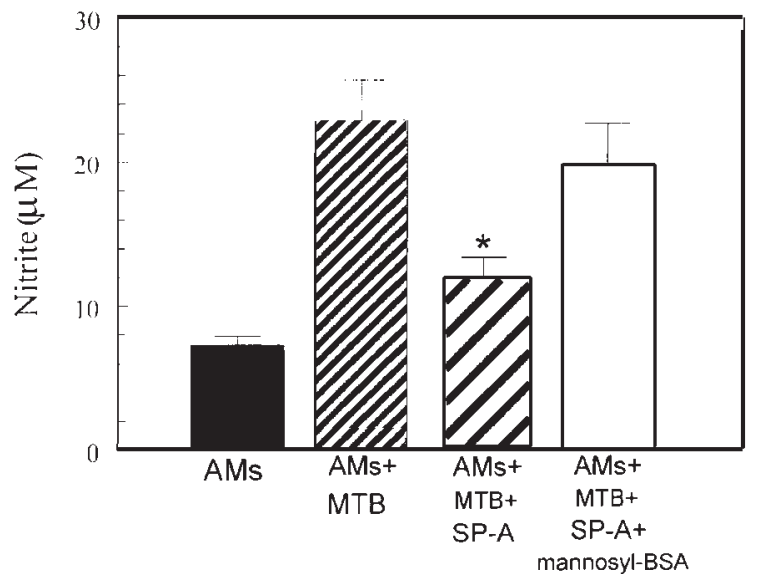

$\boldsymbol{b}$

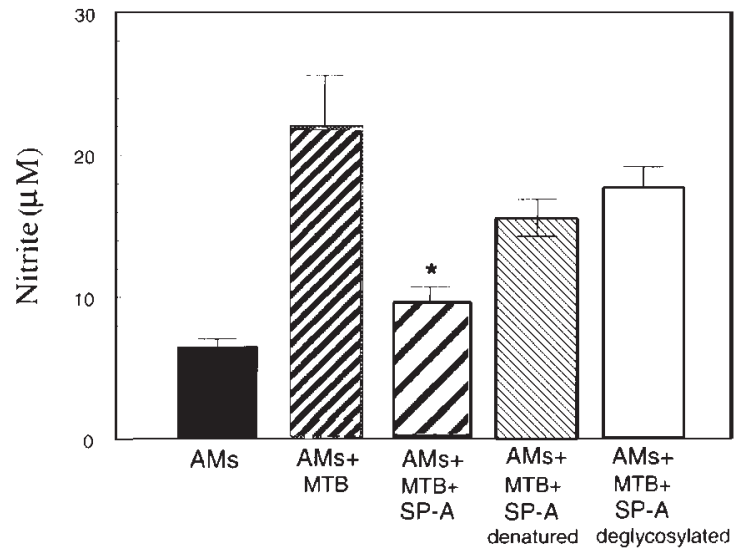

Figure 5

Effect of mannosyl-BSA, denatured SP-A, and deglycosylated SP-A on IFN- $\gamma$-induced RNI production by M. tuberculosis-infected AMs. (a) RNI production was examined after preincubation of the IFN- $\gamma$-primed AMs and $M$. tuberculosis with mannosyl-BSA (1,000 $\mu \mathrm{g}$ with a 26:1 molar ratio of mannose/albumin). Mannosyl-BSA significantly blocked the effect of SP-A on the RNI production. ( ${ }^{*} P<0.05$, comparison with either AMs $+M$. tuberculosis or AMs $+M$. tuberculosis + SP-A + mannosyl-BSA.) (b) Incubating either denatured SP-A or deglycosylated SP-A with IFN- $\gamma$-primed AMs infected with M. tuberculosis did not significantly suppress RNI production ( $P>0.05$, both comparisons). Results are expressed as mean \pm SEM for six experiments performed in triplicate.

production by IFN- $\gamma$-primed AMs incubated with $M$. tuberculosis was decreased by SP-A in a concentrationdependent manner (Fig. 2), with the maximal decrease occurring at $10 \mu \mathrm{g} / \mathrm{ml} \mathrm{SP}-\mathrm{A}(P<0.001)$. Longer incubations of AMs with M. tuberculosis and SP-A had no significant effect on RNI production.

In the presence of LPS, AMs infected with M. tuberculosis generated RNI levels of $7.0 \pm 2.4 \mu \mathrm{M}$, and the addition of SP-A to the same reaction mixture generated RNI levels of $4.0 \pm 2.4 \mu \mathrm{M}(P=0.294)$ (Fig. $3 a)$. Furthermore, there was no evidence that SP-A scavenges RNI products (Fig. $3 b$ ). Thus, the ability of SP-A to reduce RNI generation by AMs does not appear to be secondary to interference with the nitrite assay.

To determine the specificity of the SP-A effect on RNI production, the assay was performed in the presence of $100 \mu \mathrm{g} / \mathrm{ml}$ purified $\mathrm{F}\left(\mathrm{ab}^{\prime}\right)_{2}$ fragment of anti-human SPA. Addition of anti-SP-A F $\left(\mathrm{ab}^{\prime}\right)_{2}$ fragments to the mixture of SP-A, M. tuberculosis, and AMs significantly increased RNI production from $10.6 \pm 0.7 \mu \mathrm{M}$ to $18.3 \pm 1.2 \mu \mathrm{M}$ (Fig. 4). A control antibody (normal IgG) did not block the inhibitory effect of SP-A (data not shown). Both human SP-A and rat SP-A significantly inhibited RNI production from $17.5 \pm 0.8 \mu \mathrm{M}$ to $8.1 \pm 0.7 \mu \mathrm{M}$ and $8.8 \pm 0.5 \mu \mathrm{M}$, respectively $(P<0.05$ for both comparisons).

To determine possible mechanism(s) involved in the SPA-decreased RNI production, AMs and M. tuberculosis were preincubated with SP-A in the presence or absence of mannosyl-BSA. Mannosyl-BSA significantly blocked the effects of SP-A on RNI production by IFN- $\gamma$-primed AMs in response to $M$. tuberculosis (Fig. 5a). Furthermore, neither deglycosylated SP-A nor heat-inactivated SP-A (Fig. $5 b)$ had a significant effect on RNI production (17.7 \pm 1.7 $\mu \mathrm{M}$ and $16.1 \pm 1.6 \mu \mathrm{M}$, respectively) compared with control $(22.1 \pm 2.2 \mu \mathrm{M})$. These results indicate that blocking SP-A with mannosyl-BSA or anti-SP-A $\mathrm{F}\left(\mathrm{ab}^{\prime}\right)_{2}$ fragments prevents its inhibitory effect on RNI production of AMs stimulated by IFN- $\gamma$ and $M$. tuberculosis. These results suggest that the oligosaccharide component of SP-A is involved in the SP-A-mediated decrease in RNI production by AMs in response to $M$. tuberculosis infection.

Effect of SP-A on growth of M. tuberculosis in AMs. Incubation of AMs and M. tuberculosis with SP-A significantly enhanced the growth of $M$. tuberculosis organisms (expressed as growth index) from $145.6 \pm 14.0$ to $842.4 \pm$ 97.8. Addition of anti-SP-A F $\left(\mathrm{ab}^{\prime}\right)_{2}$ fragments to the mix-

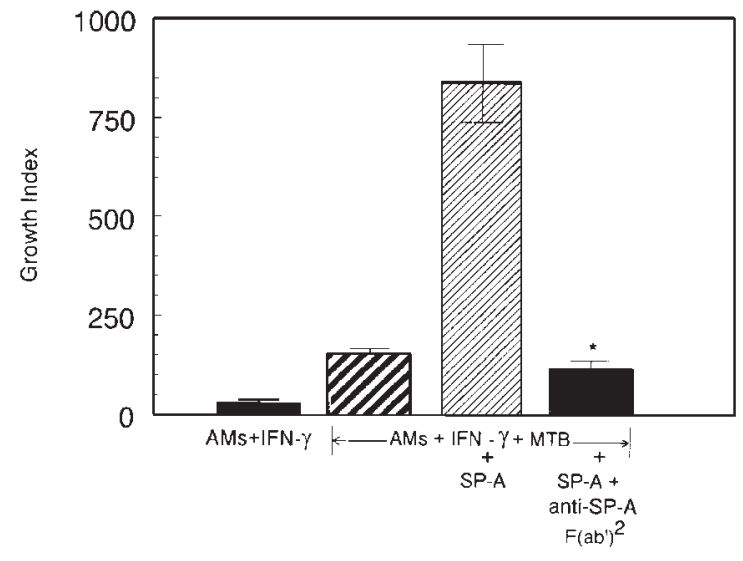

\section{Figure 6}

Effect of SP-A on M. tuberculosis growth. Effect of SP-A on M. tuberculosis growth was determined by the BACTEC radiometric method. AMs were infected with M. tuberculosis at a ratio of 10:1 (M. tuberculosis:AM) in the presence of IFN- $\gamma$ $(100 \mathrm{U} / \mathrm{ml})$, SP-A $(5 \mu \mathrm{g} / \mathrm{ml})$, or anti-SP-A antibodies $(100 \mu \mathrm{g} / \mathrm{ml})$. AMs were washed with DMEM to remove unbound $M$. tuberculosis organisms, and IFN$\gamma$, SP-A, and SP-A antibodies were added back with the fresh medium for 24 h. The AMs were then lysed and inoculated into the BACTEC vials. The vials were then incubated at $37^{\circ} \mathrm{C}$, with growth of $M$. tuberculosis being determined every $24 \mathrm{~h}$ by the BACTEC TB instrument. Preincubation of AMs and $M$. tuberculosis with SP-A significantly increased $M$. tuberculosis growth. These results suggest that blocking the effect of SP-A again restores the ability of AMs to inhibit the growth of M. tuberculosis. 
ture of SP-A, M. tuberculosis, and AMs significantly decreased the growth of M. tuberculosis from $842.4 \pm 97.8$ to $124.8 \pm 18.7$ (Fig. 6). It did not appear that SP-A had any direct effect independent of macrophages, i.e., on diminishing the toxicity of RNIs to the M. tuberculosis organisms. When M. tuberculosis organisms were grown in the absence of macrophages but in the presence of $\mathrm{NaNO}_{3}(100 \mu \mathrm{M})$, growth was decreased from $855.5 \pm$ 143.6 to $476.0 \pm 185.4$. However, this inhibitory effect of M. tuberculosis growth was not affected by the presence of SP-A (465.0 \pm 138.1$)$. Thus, SP-A did not alter the growthinhibiting effects of RNIs on M. tuberculosis.

\section{Discussion}

The results of this study suggest that SP-A suppresses RNI production in IFN- $\gamma$-treated AMs in response to $M$. tuberculosis and that the oligosaccharide component of SP-A is critical in this interaction. SP-A suppressed RNI production by IFN- $\boldsymbol{\gamma}$-stimulated AMs in a concentration-dependent manner in response to M. tuberculosis, and the inhibitory effect of SP-A could be blocked with both anti-SP-A $\mathrm{F}\left(\mathrm{ab}^{\prime}\right)_{2}$ fragments and mannosyl-BSA. Deglycosylated or heat-inactivated SP-A failed to suppress the RNI production. This suggests SP-A-mediated attachment/phagocytosis of M. tuberculosis represents one possible mechanism by which M. tuberculosis organisms can safely enter its host cell without triggering a cytotoxic response. In this way, AMs may provide a potentially safe habitat whereby the organisms are able to survive and multiply.

Recent investigations have focused on the role of RNIs in host defense against $M$. tuberculosis (22). For instance, IFN- $\gamma$ priming of L-arginine-dependent RNI production plays a major role in growth inhibition or killing of $M$. tuberculosis (23-25). Likewise, in vivo RNI production was shown to be greatly reduced in IFN- $\gamma$ knockout mice, and this resulted in unrestricted growth of M. tuberculosis (58). CD4 T lymphocytes appear to be the major source of IFN- $\gamma$ early during the course of infection with M. tuberculosis (59). The cytokine IL-12 appears to upregulate IFN- $\gamma$, whereas IL-10 downregulates IFN- $\gamma$ in response to M. tuberculosis infection $(60,61)$. In our study, AMs primed with IFN- $\gamma$ and incubated with $M$. tuberculosis markedly increased RNI production in a concentrationdependent manner, with maximum production occurring at $100 \mathrm{U}$ IFN- $\gamma / \mathrm{ml}$.

It is known that $M$. tuberculosis can interact with various AM receptors (12-18), and yet it frequently avoids being killed by the AMs. Specifically, it has been suggested that SP-A- or complement-mediated attachment/phagocytosis by AMs may avoid triggering a cytotoxic response $(36,37$, 62). Our study indicates an SP-A concentration-dependent suppression of RNI production by IFN- $\gamma$-primed AMs in response to $M$. tuberculosis. This occurs despite the fact that SP-A increases the attachment/phagocytosis of M. tuberculosis by AMs (52), resulting in AMs having higher numbers of $M$. tuberculosis organisms intracellularly.

SP-A is well recognized to be an effective innate opsonin in the alveolar spaces as an important part of innate immunity within the lungs (63). SP-A can stimulate directed chemotaxis by alveolar macrophages to microorganisms (64). Furthermore, the role of SP-A in host defense is supported by the recent study of LeVine et al. (65), which demonstrates that SP-A-deficient mice have increased susceptibility to group B streptococcal infection. In contrast to extracellular pathogens such as streptococci, mycobacterial species must enter and subsequently grow within the alveolar macrophage (4-11). Our study suggests that $M$. tuberculosis may use this SP-A-mediated entry mechanism to its own advantage.

Recent studies from our lab indicated that only the glycosylated, not the deglycosylated, form of SP-A binds to M. tuberculosis (52). Gaynor et al. (47) also showed that deglycosylated SP-A does not enhance the adherence of M. tuberculosis to monocytes. In addition, in our study, the data (Fig. 5b) suggest that deglycosylated SP-A does not inhibit the RNI production by alveolar macrophages incubated with M. tuberculosis. Therefore, the previous studies and the current data suggest that sugars in the carbohydrate recognition domain of SP-A may be important in the ability of SP-A to recognize $M$. tuberculosis and to mediate an inhibitory effect on the alveolar macrophage response.

Contrary to our current study, others have demonstrated a stimulatory influence of SP-A on AMs (39, 40, 66). The reasons why SP-A in some studies appears to stimulate $\operatorname{AMs}(39,40,66)$ and in others appears to dampen the AM response (36-38) is not clear. There is some evidence that the method of isolation and purification of SP-A may alter SP-A function (39). Also, SP-A appears to avidly bind LPS (67), and preliminary studies suggest SP-A may enhance presentation of LPS to AMs (Stamme, C.S., and Wright, J.R., unpublished data). Similar LPS-binding properties have been described for SP-D (68); thus, even a trace contamination of endotoxin with SP-A might alter the AM response. Further study is clearly needed in defining the interaction of SP-A, endotoxin, and AMs.

It is also recognized that SP-A likely functions differently in vitro than in the complex lipid-rich milieu of the alveolar spaces (69). As a result, our in vitro studies may not accurately reflect the interactions of SP-A, $M$. tuberculosis, and AMs as they occur in vivo. However, our initial study of SP-A's effect on M. tuberculosis and AMs used human BAL fluid; in contrast to culture media and artificial surfactants, the intact BAL contained at least some of the native alveolar constituents that might influence SP-A function (42). Thus, we believe the current study is relevant to the alveolar conditions that might influence the interactions of M. tuberculosis and AMs.

As might be predicted, a decrease in RNI production by AMs incubated with SP-A-coated M. tuberculosis organisms was associated with a commensurate increase in growth of M. tuberculosis within the AMs. Increased $M$. tuberculosis growth may occur for multiple reasons. It is likely that both increased numbers of $M$. tuberculosis organisms in AMs (52) and a decrease in RNI production permit increased growth $(22,23)$. Our observations suggest that elevation of SP-A in the lung may represent one reason why HIV-infected individuals may be more susceptible to M. tuberculosis infections.

Another pulmonary disorder associated with elevated levels of SP-A is silicosis (70), a condition associated with a lifelong predisposition to tuberculosis (71). The mechanisms for the elevation of SP-A in silicosis are unclear, 
but this may represent a compensatory mechanism by the lung to facilitate clearance of silica particles from the lower respiratory tract. HIV infection and silicosis are very different conditions; however, it is interesting that both are associated with elevated SP-A levels and both have an increased risk for pulmonary tuberculosis.

Further studies of the mechanisms of $M$. tuberculosis survival in the intracellular environment of the AM may provide important information regarding the earliest stages in the pathogenesis of the disease, and this may permit the development of novel therapeutic strategies to modulate the disease process.

\section{Acknowledgments}

This study was supported by National Institutes of Health grants R01 HL-51962, R01 HL-43524, and R01 HL-46647.

1. Read, C. 1996. Mycobacterium tuberculosis: a stowaway in the cell. Lancet. 348:50.

2. Raviglione, M.C., Snider, D.E., Jr., and Kocki, A. 1995. Global epidemiology of tuberculosis: morbidity and mortality of a worldwide epidemic. JAMA. 273:220-226.

3. Bloom, B.R., and Murray, C.J.L. 1992. Tuberculosis: commentary on a reemergent killer. Science. 257:1055-1063.

4. Rook, G., and Hernandez-Pando, R. 1996. The pathogenesis of tuberculosis. Annu. Rev. Microbiol. 50:259-284.

5. Mehta, P., King, C., White, E., Murtagh, J., Jr., and Quinn, F. 1996. Comparison of in vitro models for the study of Mycobacterium tuberculosis invasion and intracellular replication. Infect. Immun. 64:2673-2679.

6. Bermudez, L.E., and Goodman, J. 1996. Mycobacterium tuberculosis invades and replicates within type II alveolar cells. Infect. Immun. 64:1400-1406.

7. Fenton, M.J., and Vermeulen, M.W. 1996. Immunopathology of tuberculosis: roles of macrophages and monocytes. Infect. Immun. 64:683-690.

8. Schlesinger, L.S. 1996. Role of mononuclear phagocytes in M. tuberculosis pathogenesis. J. Invest. Med. 44:312-323.

9. Chan, J., and Kaufmann, S.H. 1994. Immune mechanisms of protection. In Tuberculosis: pathogenesis, protection and control. B. Bloom, editor. ASM Press. Washington, DC. 389-415.

10. Clemens, D.L., and Horwitz, M.A. 1995. Characterization of the Mycobacterium tuberculosis phagosome and evidence that phagosomal maturation is inhibited. J. Exp. Med. 181:257-270.

11. Via, L.A.E., et al. 1997. Arrest of mycobacterial phagosome maturation is caused by a block in vesicle fusion between stages controlled by rab5 and rab7. J. Biol. Chem. 272:13326-13331.

12. Schlesinger, L.S., Bellinger-Kawahara, C.G., Payne, N.R., and Horwitz, M.A.A. 1990. Phagocytosis of Mycobacterium tuberculosis is mediated by human monocyte complement receptors and complement component C31. J. Immunol. 144:2771-2780.

13. Schlesinger, L.S., and Horwitz, M.A. 1991. Phagocytosis of Mycobacteri um leprae by human monocyte-derived macrophages is mediated by complement receptors CR1 (CD35), CR3 (CD11b/CD18), and CR4 $(\mathrm{CD} 11 \mathrm{c} / \mathrm{CD} 18)$ and IFN- $\gamma$ activation inhibits complement receptor function and phagocytosis of this bacterium. J. Immunol. 147:1983-1994.

14. Schlesinger, L.S. 1993. Macrophage phagocytosis of virulent but not attenuated strains of Mycobacterium tuberculosis is mediated by mannose receptors in addition to complement receptors. J. Immunol. 150:2920-2930.

15. Roecklein, J.A., Swartz, R.P., and Yeager, H., Jr. 1992. Nonopsonic uptake of Mycobacterium avium complex by human monocytes and alveolar macrophages. J. Lab. Clin. Med. 119:772-781.

16. Peterson, P.K., et al. 1995. CD14 receptor-mediated uptake of nonopsonized Mycobacterium tuberculosis by human microglia. Infect. Immun. 63:1598-1602.

17. Ezekowitz, A.B., Sastry, K., Bailly, P., and Warner, A. 1990. Molecular characterization of the human macrophage mannose receptor: demonstration of multiple carbohydrate recognition-like domains and phagocytosis of yeasts in Cos-1 cells. J. Exp. Med. 172:1785-1794.

18. Schorey, J.S., Carrol, M.C., and Brown, E.J. 1997. A macrophage invasion mechanism of pathogenic mycobacteria. Science. 277:1091-1093.

19. Hirsch, C.S., Ellner, J.J., Russell, D.G., and Rich, E.A. 1994. Complement receptor-mediated uptake and tumor necrosis factor-mediated growth inhibition of Mycobacterium tuberculosis by human alveolar macrophages. J. Immunol. 152:743-753.

20. Fels, A.O., and Cohn, Z.A. 1986. The alveolar macrophage. J. Appl. Physiol. 60:353-369.

21. Ding, A.H., Nathan, C.F., and Stuehr, D.J. 1988. Release of reactive nitrogen intermediates and reactive oxygen intermediates from mouse peri- toneal macrophages: comparison of activating cytokines and evidence for independent production. J. Immunol. 141:2407-2413.

22. Denis, M. 1991. Interferon-treated murine macrophages inhibit growth of tubercle bacilli via the generation of reactive nitrogen intermediates cell. Cell. Immunol. 132:150-157.

23. Chan, J., Xing, Y., Magliozzo, R.S., and Bloom, B.R. 1992. Killing of virulent Mycobacterium tuberculosis by reactive nitrogen intermediates produced by activated murine macrophages. J. Exp. Med. 175:1111-1122.

24. Flesch, I.E., and Kaufmann, S. 1991. Mechanisms involved in mycobacterial growth inhibition by interferon-activated bone marrow macrophages: role of reactive nitrogen intermediates. Infect. Immun. 59:3213-3218.

25. Flynn, J.L., et al. 1993. An essential role for interferon- $\gamma$ in resistance to Mycobacterium tuberculosis infection. J. Exp. Med. 178:2249-2254.

26. Greenburg, S., Xie, J., Kolls, J., Mason, C., and Didier, P. 1995. Rapid induction of mRNA for nitric oxide synthase II in rat alveolar macrophages by intratracheal administration of Mycobacterium tuberculosis and Mycobacterium avium. Proc. Soc. Exp. Biol. Med. 209:46-53.

27. MacMaking, J., et al. 1997. Identification of a nitric oxide synthase as a protective locus against tuberculosis. J. Immunol. 94:5243-5248.

28. McCormack, F. 1997. The structure and function of surfactant proteinA. Chest. 111:114s-119s.

29. Hawgood, S. 1989. Pulmonary surfactant apoproteins: a review of protein and genomic structure. Am. J. Physiol. 257:L13-L21.

30. Hawgood, S., and Shiffer, K. 1991. Structures and properties of the surfactant-associated proteins. Annu. Rev. Physiol. 53:375-394.

31. Voss, T., Eistetter, H., Schafer, K., and Engel, J. 1988. Macromolecular organization of natural and recombinant lung surfactant protein SP 2836. J. Mol. Biol. 201:219-227.

32. Tenner, A., Robinson, S., Borchelt, J., and Wright, J.R. 1989. Human pulmonary surfactant protein (SP-A), a protein structurally homologous to C1q, can enhance FcR- and CR1-mediated phagocytosis. J. Biol. Chem. 264:13923-13928.

33. van Iwaarden, J.F., et al. 1991. Surfactant protein A is opsonin in phagocytosis of herpes simplex virus type 1 by rat alveolar macrophages. Am. J. Physiol. 261:L204-L209.

34. McNeely, T.B., and Coonrod, J.D. 1994. Aggregation and opsonization of type A but not type B Hemophilus influenzae by surfactent protein A. Am. J. Respir. Cell Mol. Biol. 11:114-122.

35. Bobak, D.A., Gaither, T.A., Frank, M.M., and Tenner, A.J. 1987. Modulation of FCR function by complement: subcomponent $\mathrm{C} 1 \mathrm{q}$ enhances the phagocytosis of IgG-opsonized targets by human monocytes and culture-derived macrophages. J. Immunol. 138:1150-1156.

36. Katsura, H., Kawada, H., and Konno, K. 1993. Rat surfactant apoprotein A (SP-A) exhibits antioxidant effects on alveolar macrophages. Am. J. Respir. Cell Mol. Biol. 9:520-525.

37. Weber, H., Heilmann, P., Meyer, B., and Maier, K.L. 1990. Effect of canine surfactant protein (SP-A) on the respiratory burst of phagocytic cells. FEBS Lett. 270:90-94.

38. Borron, P., et al. 1996. Surfactant protein-A inhibits human lymphocyte proliferation and IL-2 production. Am. J. Respir. Cell Mol. Biol. 15:115-121.

39. van Iwaarden, J.F., Teding Van Berkout, F., Whitsett, J.A., Oosting, R.S., and van Golde, L.M. 1995. A novel procedure for the rapid isolation of surfactant protein A with retention of its alveolar-macrophage-stimulating properties. Biochem. J. 309:551-555.

40. van Iwaarden, F., Welmers, B., Verhoef, J., Haagsman, H.P., and van Golde, L.M. 1990. Pulmonary surfactant protein A enhances the hostdefense mechanism of rat alveolar macrophages. Am. J. Respir. Cell Mol. Biol. 2:91-93.

41. Barnes, P.F., Bloch, A.B., Davidson, P.T., and Snider, D.E., Jr. 1991. Tuberculosis in patients with human immunodeficiency virus infection. $N$. Engl. J. Med. 324:1644-1650.

42. Downing, J.F., Pasula, R., Wright, J.R., Twigg, H.L., III, and Martin, W.J., II. 1995. Surfactant protein A promotes attachment of Mycobacterium tuberculosis to alveolar macrophages during infection with HIV. Proc. Natl. Acad. Sci. USA. 92:4848-4852.

43. Dhople, A., Dhople, A., and Ibanzez, M. 1995. In vitro activities of 2,2'bipyridyl analogues against Mycobacterium avium and Mycobacterium tuberculosis. Tuber. Lung Dis. 76:136-140.

44. Wright, J.R., Wager, R.E., Hawgood, S., Dobbs, L., and Clements, J.A. 1987. Surfactant apoprotein $M_{r}=26,000-36,000$ enhances uptake of liposomes by type II cells. J. Biol. Chem. 262:2888-2894.

45. Lowry, O.H., Rosebrough, N.J., Farr, A.L., and Randall, R.J. 1951. Protein measurement with the folin phenol reagent. J. Biol. Chem. 193:265-275.

46. Laemmli, U. 1970. Cleavage of structural proteins during the assembly of the head of bacteriophage T4. Nature. 227:680-685.

47. Gaynor, C., McCormack, F.X., Voelker, D.R., McGowan, S.E., and Schlesinger, L.S. 1995. Pulmonary surfactant protein A mediates enhanced phagocytosis of Mycobacterium tuberculosis by a direct interaction with human macrophages. J. Immunol. 155:5343-5351.

48. Kuroki, Y., Mason, R.J., and Voelker, D.R. 1988. Alveolar type II cells express a high-affinity receptor for pulmonary surfactant protein A. Proc. 
Natl. Acad. Sci. USA. 85:5566-5570.

49. McIntosh, J., Swyers, A., Fisher, J., and Wright, J. 1996. Surfactant proteins $\mathrm{A}$ and $\mathrm{D}$ increase in response to intratracheal lipopolysaccharide. Am. J. Respir. Cell Mol. Biol. 15:509-519.

50. Kohler, G., and Milstein, C. 1975. Continuous cultures of fused cells secreting antibodies of predefined specificity. Nature. 256:495-497.

51. de Blas, A.L., Ratnaparkhi, M.V., and Mosimann, J.E. 1983. Estimation of the number of monoclonal hybridomas in a cell-fusion experiment. Methods Enzymol. 92:36-39.

52. Pasula, R. et al. 1997. Surfactant protein A (SP-A) mediates attachment of Mycobacterium tuberculosis to murine alveolar macrophages. Am. J. Respir. Cell Mol. Biol. 17:209-217.

53. Green, L.C., et al. 1982. Analysis of nitrite, nitrite and $\left[{ }^{15} \mathrm{~N}\right]$ nitrite in biological fluids. Anal. Biochem. 126:131-138.

54. Raetz, C., et al. 1991. Gram-negative endotoxin: an extraordinary lipid with profound effects on eukaryotic signal transduction. FASEB J. 5:2652-2660.

55. Reddy, M.V., Srinivasan, S., Andersen, B., and Gangadharam, P.R.J. 1994. Rapid assessment of mycobacterial growth inside macrophages and mice, using the radiometric (BACTEC) method. Tuber. Lung Dis. 75:127-131.

56. Kirihara, J.M., Hillier, S.L., and Coyle, M.B. 1985. Improved detection times for Mycobacterium avium and Mycobacterium tuberculosis with the BACTEC radiometric system. J. Clin. Microbiol. 22:841-845.

57. Kuebler, R.R., and H. Smith. 1976. Statistics. John Wiley and Sons. New York, NY. 175-274.

58. Cooper, A.M., et al. 1993. Disseminated tuberculosis in interferon gamma gene-disrupted mice. J. Exp. Med. 178:2243-2247.

59. Orme, I.M., Roberts, A.D., Griffen, J.P., and Abrams, J.S. 1993. Cytokine secretion by $\mathrm{CD} 4 \mathrm{~T}$ lymphocytes acquired in response to Mycobacterium tuberculosis infection. J. Immunol. 151:518-525.

60. Flynn, J.L., et al. 1995. IL-12 increases resistance of BALB/c mice to
Mycobacterium tuberculosis infection. J. Immunol. 155:2515-2524.

61. Gong, J.H., et al. 1996. Interleukin-10 downregulates Mycobacterium tuberculosis-induced Th1 responses and CTLA-4 expression. Infect. Immun. 64:913-919.

62. Silverstein, S.C., Greenberg, S., Di Virgilio, F., and Steinberg, T.H. 1989. Phagocytosis. In Fundamental immunology. W.E. Paul, editor. Raven Press. New York, NY. 703-720

63. Pison, U., Max, M., Neuendank, A., Weissbach, S., and Pietschmann, S. 1994. Host defence capacities of pulmonary surfactant: evidence for 'non-surfactant' functions of the surfactant system. Eur. J. Clin. Invest. 24:586-599.

64. Wright, J.R., and Youmans, D.C. 1993. Pulmonary surfactant protein A stimulates chemotaxis of alveolar macrophage. Am. J. Physiol. 264:L338-L344.

65. LeVine, A.M., et al. 1997. Surfactant protein A-deficient mice are susceptible to group B streptococcal infection. J. Immunol. 158:4336-4340.

66. Blau, H., Riklis, S., Van Iwaarden, J.F., McCormak, F.X., and Kalina, M. 1997. Nitric oxide production by rat alveolar macrophages can be modulated in vitro by surfactant protein A. Am. J. Physiol. 16:L1198-L1204.

67. Kalina, M., Blau, H., Riklis, S., and Kravtsov, V. 1995. Interaction of surfactant protein A with bacterial lipopolysaccharide may affect some biological functions. Am. J. Physiol. 268:L144-L151.

68. Kuan, S.-F., Rust, K., and Crouch, E. 1992. Interactions of surfactant protein D with bacterial lipopolysaccharides. J. Clin. Invest. 90:97-106.

69. Childs, R., et al. 1992. Specificity of lung surfactant protein SP-A for both the carbohydrate and the lipid moieties of certain neutral glycolipids. $J$. Biol. Chem. 267:9972-9979.

70. Lesur, O., et al. 1993. Surfactant associate proteins (SP-A, SP-B) are increased proportionally to alveolar phospholipids in sheep silicosis. Lung. 171:63-74.

71. Snider, D.E., Jr. 1978. The relationship between tuberculosis and silicosis. Am. Rev. Respir. Dis. 118:445-460. 